\title{
COMPUTER SIMULATIONS OF WATER AND AQUEOUS ELECTROLYTE SOLUTIONS AT INTERFACES*
}

\author{
E. SPOHR and K. HeINZINGER
}

\begin{abstract}
Max-Planck-Institut für Chemie (Otto-Hahn-Institut), D-6500 Mainz, Federal Republic of Germany
\end{abstract}
(Received 18 January 1988)

\begin{abstract}
The results of computer simulations of water and aqueuus electrolyte solutions in contact with smooth and corrugated walls are reviewed. Oxygen atom density profiles have been calculated by various authors from simulations using different water models in contact with hard walls, Lennard-Jones type walls of various powers, and polarizable walls where the polarizability is described by image charges. These results for smooth walls are compared with the density profiles obtained for the vicinity of a corrugated platinum crystal. There is general agreement that the dipole vectors of the water molecules near the walls are preferentially oriented parallel to the interface. Dipole distribution functions are discussed with respect to the electrostatic surface potential drop. The inhomogeneities and anisotropies of water-water pair correlation functions near smooth and corrugated walls are presented in detail. The hydration shells of ions at interfaces differ from those in the bulk solutions as demonstrated by a simulation of a 2.2 molal Lil solution between Lennard-Jones walls. Finally, the self-diffusion coefficients, the hindered translational motions of the water molecules parallel and perpendicular to smooth and corrugated walls, and the librational motions are discussed, calculated from the simulations with the help of the corresponding velocity autocorrelation functions.
\end{abstract}

\section{INTRODUCTION}

The investigation of the influence of non-polar walls on the structural properties of liquid water by computer simulations has received increasing interest in recent years. Jönsson[1] used in his Monte Carlo (MC) simulations the MCY model for water[2] to study the influence of rigid walls while Christou et al.[3] investigated the interactions of 9-3 Lennard-Jones (LJ) walls with liquid water employing the Rowlinson model[4]. Marchesi [5] reported results of a Molecular Dynamics (MD) simulation with the ST2 model of water[6] where the water-wall interactions were described by a purely repulsive potential. Another MD study with the ST2 model was performed by Sonnenschein and Heinzinger [7] where a 12-6 LJ potential was employed for the water-wall interactions. Barabino et al.[8] reported MD simulations with the MCY model for water and a 4-2 LJ potential with image charges for the water-wall potential, while Lee et al.[9] employed the ST2 model and a 9-3 potential. The MC simulations of Christou et al. [10] with Rowlinson water between hard walls were performed for a density of $1.5 \mathrm{~g} \mathrm{~cm}^{-3}$. More recently the TIPS2 model[11] has been employed in MC studies of water between 9-3 LJ walls by Aloisi et al.[12] and hard walls by Valleau and Gardner[13]. To this category of investigations belongs also the MC study of a benzene-water interface with the MCY model for water by Linse[14].

Only one simulation of an aqueous electrolyte solution between walls has been reported so far. Spohr and Heinzinger[15] investigated by MD a 2.2 molal

* Presented at the Conference on the Structure of Liquids and Solutions held at Veszprém. Hungary, 24-27 August 1987.
LiI solution with the ST2 model for water between 12$6 \mathrm{LJ}$ walls. The effect of the walls is demonstrated by comparison with the results of an MD simulation of a bulk Lil solution at the same concentration[16-18].

The first simulation of water near a polar interface was reported by Anastasiou et al.[19]. These authors performed MD simulations with the CF model for water[20] at the surface of an insoluble $\mathrm{NaCl}$ crystal. In the MD simulations of Kjellander and Marcelja[21, 22] with the ST2 model for water the walls represent a mineral and a lecithin surface, where the atoms bear elementary charges and the polar groups partial charges in addition to $\mathrm{LJ}$ interactions between the water molecules and the atoms of the walls.

The investigation of water-metal interfaces by computer simulations started just recently with an MD simulation of ST2 water between platinum walls[23]. The water molecules interact with the $(100)$ face of a platinum crystal slab consisting of 550 atoms. Again the ST2 model has been employed in two MC studies of water between metal plates by Parsonage and Nicholson $[24,25]$. The water-wall interactions are described by image charges and a 9-3 LJ potential with the parameters chosen in such a way that they represent a neon atom interacting with a copper surface. Finally, two further MC studies are reported by Gardner and Valleau[26] with the TIPS2 model for water between hard walls. In both cases one wall was non-polar while the second one was either charged or metallic (image charges).

Besides the simulations with realistic water models between polar and non-polar walls, model fluids like hard spheres with imbedded dipoles between hard walls have also been investigated $[27,28]$. These model fluids have the advantage that they can be treated both by simulation and analytically. The results achieved for 
the double layer with the Primitive Model have been reviewed recently by Carnie[29] while Rosinberg et al. $[30]$ reported on the progress for the non-primitive models.

In this review we shall concentrate on $\mathrm{MD}$ and $\mathrm{MC}$ simulations of water and electrolyte solutions near planar non-polar and metallic walls. After a short presentation of the details of the simulations in section 2 , we shall discuss in section 3 the relationship between potentials and density profiles. In section 4 the distributions of water molecule orientations will be presented, followed by the effect of the walls on the water pair correlation functions in section 5 . In section 6 the differences in translational and rotational $\mathrm{dy}$ namics between smooth and corrugated surfaces are analysed.

\section{SIMULATIONS}

In all cases discussed in this review the water molecules (or the electrolyte solution) are confined to a square prism with the walls placed at the quadratic sides. In the directions parallel to the wall ( $x$ - and $y$ directions) periodic boundary conditions are applied. Figure 1 shows as example the prism employed for the simulations of water confined by a platinum crystal[23]. In all simulations except $[3,10]$ the density was chosen to be $1 \mathrm{~g} \mathrm{~cm}^{-3}$. Therefore, the dimensions of the prism depend on the number of water molecules and range from 13 up to $20 \AA$ in the $x$-and $y$-directions with distances of about 20 to $40 \AA$ between the walls. Various water models are employed in the simulations. The walls are either assumed to be hard or the water-wall interactions are described by $\mathrm{LJ}$ potentials. Additionally, image charges are used to mimic metallic walls. Some characteristic features of the various simulations are listed in Table 1.

In some simulations special features are introduced which are not listed in Table 1. Gardner and
Valleau[26] eg made only one wall polarizable (image charges) and left the other non-polar. They also investigated the effect of a charged wall. An additional

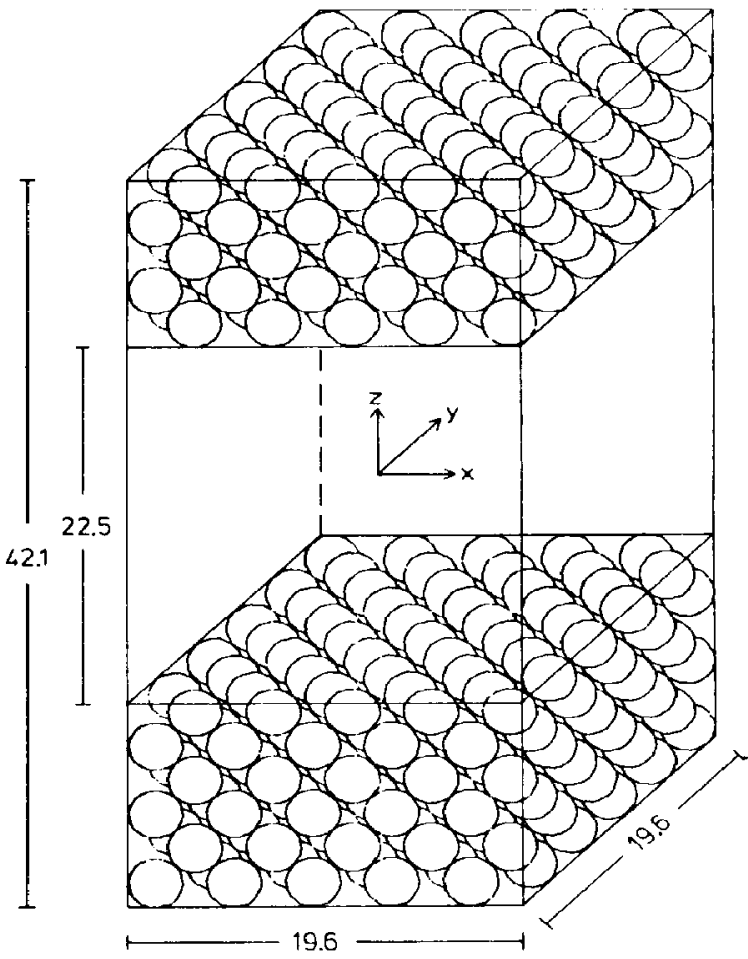

Fig. 1. Sketch of the basic tetragonal cell used in a simulation of a water lamina between two $(100)$ faces of a platinum crystal. The water molecules are located in the centre of the box and the platinum atoms are represented by the circles. All distances are in $A[15]$.

Table 1. Some characteristic data of computer simulations of water at interfaces. $T$ is the temperature, $N$ is the number of water molecules, $t$ the simulation time in ps for MD or the number of steps in $M$ passes for $M C$ simulations

\begin{tabular}{|c|c|c|c|c|c|c|}
\hline Method & $T / \mathrm{K}$ & $N$ & $t$ & Water model & Wall model & Ref. \\
\hline MC & 300 & 150 & 3.5 & MCY & hard & [1] \\
\hline MC & 298 & 216 & 2.5 & Rowlinson & 9.3 LJ, high density & {$[3]$} \\
\hline MD & 302 & 150 & 25 & ST2 & exponential & [5] \\
\hline MD & 287 & 216 & 20 & ST2 & $12-6 \mathrm{LJ}$ & [7] \\
\hline MD & 298 & 150 & $14(24)$ & MCY & hard, 4-2 LJ (+ image) & {$[8]$} \\
\hline MD & 290 & 216 & 72 & ST2 & 9-3 LJ & [9] \\
\hline MC & 363 & 216 & - & Rowlinson & hard, high density & {$[10]$} \\
\hline MC & 298 & 396 & 0.4 & TIPS2 & $9-3 \mathrm{LJ}$ & [12] \\
\hline $\mathbf{M C}$ & 399 & 125 & 1.5 & TIPS2 & hard & {$[13]$} \\
\hline MD & 283 & $200+2 \times 8$ & 10 & ST2/LiI & $12-6 \mathrm{LJ}$ & {$[15]$} \\
\hline MD & 294 & 216 & 16.4 & ST2 & $\operatorname{Pt}(100): \mathrm{LJ}+$ image & {$[23]$} \\
\hline MD & 298,323 & 216,432 & $17.5,15.5$ & ST2 & $9-3 \mathrm{LJ}+$ image $(\mathrm{Cu})$ & {$[24,25]$} \\
\hline MC & 399 & 125 & 2 & TIPS2 & hard + image & [26] \\
\hline MD & 296 & 216 & 12 & BJH & Pt(100): ext. Hückel & {$[37]$} \\
\hline
\end{tabular}


simulation by Barabino et al.[8] was performed with the assumption of a dielectric constant $\varepsilon=3$ of the wall. This is equivalent to image charges with a value of half the real charge. The simulations by Parsonage and Nicholson $[24,25]$ include calculations where the image charges are twice as high as the original ones. These authors also investigated the effect of a reaction field as an approximation of the long range Coulombic interactions. Valleau and Gardner[13] performed a series of simulations at various temperatures and concluded that at room temperature equilibrium in the density profiles cannot be reached. In the simulation of the 2.2 molal LiI solutions the ions are described by $\mathrm{LJ}$ spheres with an elementary charge at the center[15]. For further details of the various simulations the reader is referred to the original communications cited.

\section{DENSITY PROFILES}

In Fig. 2 the oxygen atom density profiles are depicted from simulations where the walls are assumed to be hard $[8,13]$. The profile reported by Jönsson [1] is quite similar to the one found by Barbino et al. [8] and is, therefore, not shown here. Also the result of the MD study with exponentially repulsive walls [5] is included as it was claimed that the profiles do not significantly depend on the softness of the repulsion. Valleau and Gardner[13] have performed their MC studies at various temperatures and did not find a strong temperature dependence of the height of the first peak. From the differences in the density profiles near the two walls they conclude that in spite of about $2 \times 10^{6}$ configurations equilibrium has not been achieved at room temperature. Therefore, their result for $399 \mathrm{~K}$ is shown in Fig. 2 (full line). It is similar to Marchesi's profile[5] at $302 \mathrm{~K}$ (dash-dotted line).

The very high peaks in the $O$-density profiles by Jönsson[1] (not shown in Fig. 2) and by Barabino et al. [8] (dotted) are difficult to understand. As it is hard to believe that the different water models used in the three simulations should be responsible for the dif-

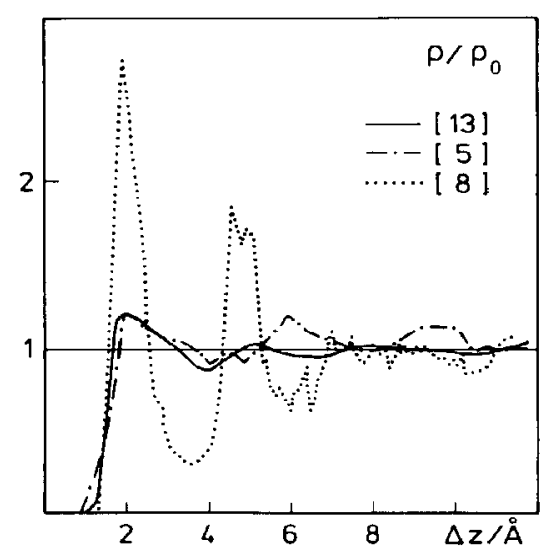

Fig. 2. Normalized oxygen atom density profiles from simulations of water between hard walls, taken from $[5,8,13]$. The density profiles have been shifted such that the first maxima are found at the same value of $\Delta z$ for all simulations. ference, it might have to be attributed to insufficient equilibration. It should be noted that the density profiles reported by Barabino et al. for other water-wall interaction models deviate also remarkably from the results of other authors using similar interaction potentials (see below).

In Fig. 3 the oxygen atom density profiles for four simulations with LJ walls are shown together with the corresponding water-wall potentials. Here it is evident how the height of the first peak depends on the depth of the potential minimum. There is no density maximum near the wall in the simulation by Sonnenschein and Heinzinger [7] because of weak interactions (dashdotted line). The differences existing between the two simulations with the 9-3 LJ potential (full and dashed lines) might have to be attributed to the different water models used in these simulations.

In the center of the lamina all four simulations show nearly bulk water density. Only for the deep water-wall potential (4-2 LJ) a second peak in the oxygen atom density profile appears (dotted line). But some doubt remains on its significance as the height of the first peak already appears to be exaggerated compared to the depth of the potential minimum (see also Fig. 2). All other oscillations-maybe with the exception of the first minimum - seem to result from the fact that the simulations are too short for a complete equilibration at room temperature as indicated by the differences in the profiles near the two walls $[7,13]$. The significantly larger box size

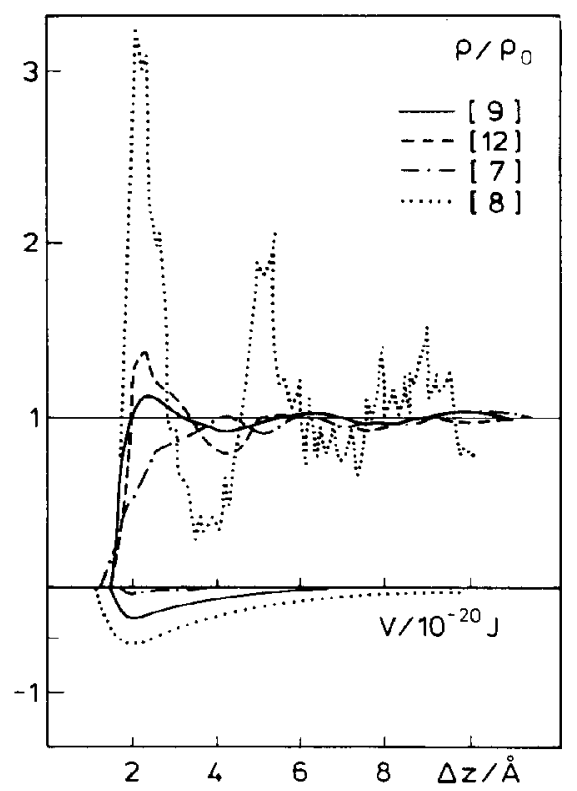

Fig. 3. Normalized oxygen atom density profiles from simulations of water between $\mathrm{LJ}$ walls (top), taken from -refs. $[7-9,12]$. The density in the bottom the water-wall interaction energies are given as a function of distance from the wall for the energetically most favourable orientation of a molecule relative to the wall. $\ln [9]$ and [12] the same interaction potential (full line) has been used (see also caption of Fig. 2). 
(wall-wall distance $38 \AA$ ) in the simulation of Aloisi et al.[12] leads-except for statistical noise - also to a constant density beyond about $6 \AA$.

Figure 4 shows the three density profiles calculated from simulations with smooth polarizable walls. The polarizability is described by means of image charge interactions which are superimposed on onedimensional 9-3 (dashed line;[24]) and 4-2 (dotted line;[8]) LJ potentials and a hard wall[26]. The absolute value of the image charges depends upon the assumed relative dielectric permittivity $\varepsilon$ which is 3 in the case of the dotted curve and $\infty$ for the remaining two curves. As can be seen from the water-wall potentials in the lower half of the figure the strength of the image charge interactions depends strongly on the position of the image plane relative to the distance of closest approach as given by the non-electrostatic interactions.

The amplitude of density oscillations for the model copper surface (dashed line) is very small even when compared with the results for unpolarizable walls (Figs 2 and 3 ). However, the oscillations range quite far. The reason for this might be found in the fact that the simulation by Parsonage and Nicholson[24] was started from an ice lattice the original structure of which did not vanish in the course of the simulation due to an insufficient number of Monte Carlo moves.

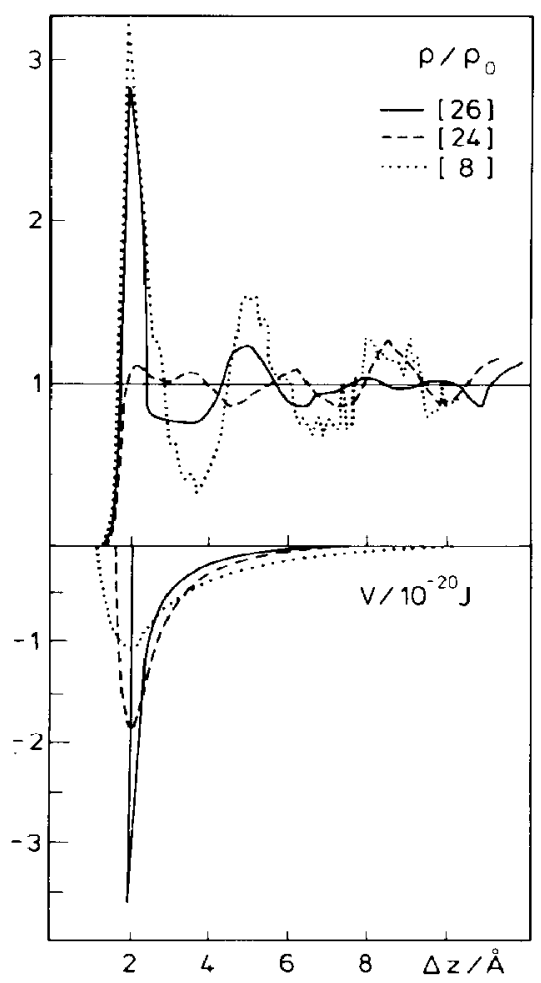

Fig. 4. Normalized oxygen atom density profiles and water-wall interaction energies from simulations of water between smooth polarizable walls, taken from $[8,24,26]$ (see also caption of Fig. 3).
Gardner and Valleau[26] (full line) find a very narrow first peak with a density increase by nearly a factor of 3 relative to the bulk density. This is in accord with the deep and narrow potential well employed in this simulation. Again a very high first maximum and two more maxima are found in the simulation by Barabino et $\mathrm{al}$.[8] (dotted line) although they use the weakest of the three water-wall interaction potentials. The reason that this group finds generally too pronounced density oscillations (see above) might result from the fact that (as in [24ך) their starting configuration was also an ice lattice.

Up to now only one simulation of water in contact with a corrugated metal wall has been reported $[23,31]$. The oxygen atom density profile near the (100) face of a platinum crystal is shown in the upper part of Fig. 5 after symmerization. The potential minimum is about twice as deep as the onc in the simulation by Gardner and Valleau[26] which represents the deepest potential well for a smooth surface. The minimum energy of $-40.3 \mathrm{~kJ} \mathrm{~mol}^{-1}$ for an isolated water molecule is at the lower end of the range of experimentally determined adsorption energies of water molecules on transition metal surfaces. These range from about -40 to about -70 $\mathrm{kJ} \mathrm{mol}^{-1}[32-35]$. The narrow peak-its height corresponds roughly to seven times the bulk densityindicates a very tight binding of the water molecules to the adsorption sites on the platinum surface. From the integral over the first peak a coverage of about $88 \%$ of the adsorption sites is calculated. Hence, on the (100) plane a perturbed monolayer is formed. Due to the tightly bonded layer of adsorbed molecules the packing effects lead to a pronounced second maximum. Beyond this maximum the density is constant but does

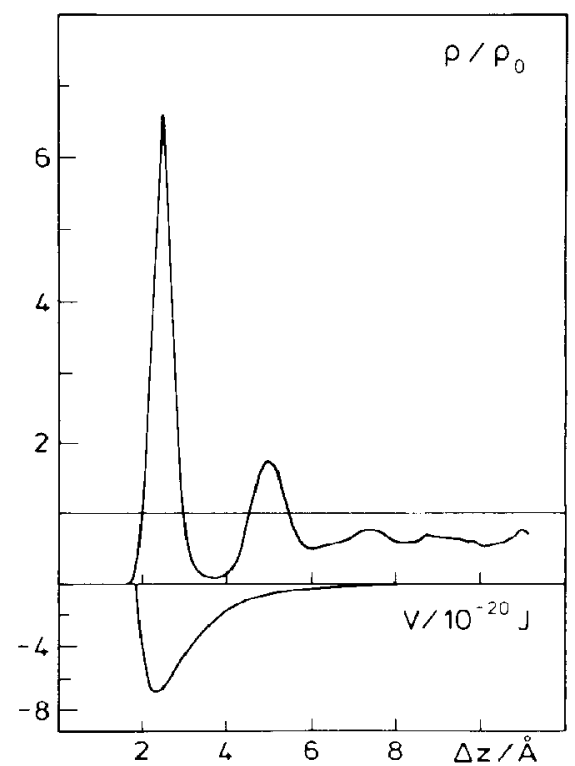

Fig. 5. Normalized oxygen atom density profile and water-wall interaction potential for a simulation of ST2 water between corrugated Pt(100) surfaces[23, 31]. 
not reach the bulk value because initially the average density of the lamina was set equal to $1.0 \mathrm{~g} \mathrm{~cm}^{-3}$.

The maxima and minima in the hydrogen atom density profiles are less pronounced than in the oxygen ones and their positions are more sensitive to the water models and the water-wall interactions employed in the simulations. The relative positions of the peaks in the oxygen and hydrogen atom density profiles depend on the orientation of the water molecules. As this information can be derived from the simulation directly (see the following chapter) the hydrogen atom profiles will not be discussed here. For details of these profiles the reader is referred to the original communications.

From the simulations of water near non-polar as well as polarizable walls discussed here it can be concluded that there is qualitative agreement as far as the relation between the height of the oxygen and hydrogen atom density profiles and the depth of the water-wall potentials is concerned. It seems to be that the simulations can provide a reliable picture of the water structure near a given wall provided the correct water-wall potential can be deduced from other sources. It might be advisable to have larger box sizes and longer simulations for more quantitative results together with an improved water-water potential (eg a flexible model).

\section{ORIENTATIONS}

In all simulations reported so far the water molecules in the wall layer are orientated preferentially with the dipole moment parallel to the surface. The extent of this ordering varies with the potentials employed. In most cases the polarization is small and cannot be determined reliably due to statistical noise.

While the preference for a dipole orientation parallel to the interfacial plane is generally agreed upon, the results on the degree of orientational anisotropy in the second and subsequent laycrs scatter significantly. Lee et al.[9] showed that the orientational distribution of water dipoles and $\mathrm{OH}$ vectors as a function of distance from the wall can be explained by assuming a thermally disturbed ice Ih lattice the $c$-axis of which is perpendicular to the surface plane. Sonnenschein and Heinzinger[7] found a general preference for the parallel orientation throughout a lamina of about 6 layers of water molecules. Valleau and Gardner[13] assumed, based on similar results, that a 6 layer lamina might be too thin in order to effectively decouple the two interfaces. However, they also found persisting orientational order in a lamina of twice the thickness and even in bulk water when the final lamina configuration was used as a starting configuration. In contrast, Marchesi[5] and Aloisi et al.[12], for example, reported orientational isotropy with respect to the normal direction in the central part of the lamina. In the system ST2 water/ $\mathrm{Pt}(100)$ [31] where the external perturbation is much stronger than in all other reported cases, isotropy has been found from the third layer on. The reported results are in agreement with the experience according to which single particle properties can depend strongly on the initial conditions. In the work on ST2 water/Pt(100) the total dipole moment was set equal to zero for each layer separately before the

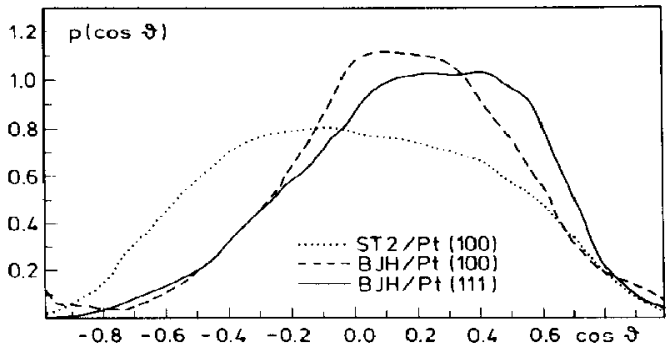

Fig. 6. Distribution of $\cos \theta$ from three simulations of water near corrugated platinum surfaces. $\theta$ is the angle between the dipole moment vector of a water molecule and the inward directed surface normal[36].

system was equilibrated completely.

Most of the water-wall potentials used do either not $[7,9,12]$ or only weakly $[1,5,8,13,24,25]$ depend on the water molecule orientations. Therefore, in all cases of weak interactions the distributions are symmetric around $\cos \theta=0$, where $\theta$ is the angle between the dipole moment dircetion of a water molecule and the vector perpendicular to the surface and pointing into the liquid. The same result has been found from a simulation with a structured and strongly adsorbing wall[23, 31,36]. Although the ST2 model with its slightly distorted tetrahedral charge distribution has been employed here, the image forces do not produce a significant orientational anisotropy (dotted curve in Fig. 6). Only Nicholson and Parsonage find a net polarization using the ST2 model together with image charge interactions near a smooth model copper surface $[24,25]$. However, there is strong noise due to insufficient sampling. As they find similar results for several simulations this result may be due to the special choice of boundary conditions (modified reaction field).

In a recent work by Spohr[37] an improved water-platinum potential model together with a flexible water model[38] was used, in which-in accordance with theoretical[39-42] and experimental $(e g[35,43.47])$ work on the binding status of water on metals - the water dipole points away from the surface for the isolated molecule. Even with this potential, preferential orientations of the water dipoles parallel to the surface are observed[36]. Figure 6 shows the distribution of water orientations with respect to the surface for $\mathrm{H}_{2} \mathrm{O}$ molecules adsorbed on a $\mathrm{Pt}(100)$ (dashed line) and on a Pt(111) (full line) crystal. The center of the distribution for the more realistic water-wall potentials is shifted towards positive values of $\cos \theta$, thus producing a net dipole moment. This leads to a solvent contribution to the interfacial potential drop according to:

$$
\chi(z)=\frac{1}{\varepsilon_{0}} \int_{0}^{x}\left\langle\mu_{z}(z)\right\rangle \rho(z) \mathrm{d} z,
$$

where $\mu_{z}$ is the $z$-component of the dipole moment and $\rho(z)$ the oxygen atom density. Averaging over the two interfaces yields values of the potential drop of $\approx$ $-0.7 \pm 0.2 \mathrm{~V}$ on the $(100)$ face and $\approx-0.9 \pm 0.2 \mathrm{~V}$ on the (111) face and is mostly due to the ordered adsorbed layer. The measured work function change 
on platinum upon monolayer adsorption of water at $100 \mathrm{~K}$ amounts to about $-1.0 \mathrm{eV}$ and upon multilayer adsorption to about $-1.2 \mathrm{eV}[43]$. Hence, both simulation and experiments agree on the dominant role of the contact layer for the surface potential drop. Furthermore, as the experimental values contain an additional contribution due to electronic polarization of the metal, the simulated values should be smaller than the experimental ones in agreement with the numbers given above.

In concluding this chapter it can be stated that there is general agreement between the various simulations as far as a preferential orientation of the dipole moments of the water molecules in the adsorbed layer parallel to the walls is concerned. There is no reliable information on the extent of this ordering, its dependence on the water-wall potentials and the distance from the wall up to which the orientation of the water molecules is influenced. In order to clarify these points longer simulations with larger numbers of water molecules are needed where special care should be taken in the setup of the initial configurations. The simulations of BJH water near a platinum surface reproduce the measured work function changes qualitatively.

\section{PAIR CORRELATION FUNCTIONS}

Several authors $[3,5,15,24,25,48,49]$ discussed atom-atom correlation functions of water near smooth walls. In order to get a deeper insight into the water structure near interfaces, the laminas are subdivided into several layers of about one or two Angstrom thickness and the radial distribution functions (RDFs) are calculated separately for reference atoms located in a given layer. While in most cases RDFs are based on the total available space around a given atom (ie all particles within a spherical shell between distance $r$ and $r+\mathrm{d} r$ contribute to $g_{\alpha \beta}(r)$ ), Marchesi[5] confined the sampling to sheets of one layer thick ness. In general, an increase in the height of the first maximum of the oxygen-oxygen RDF relative to bulk water is found. In addition, in some cases a significant decrease of the first minimum of $g_{\text {oo }}(r)$ can be seen $[5,49]$. Furthermore, Nicholson and Parsonage $[24,25]$ report an increase in the height of the second maximum in $g_{\mathrm{OH}}(r)$ which indicates a larger number of hydrogen bonds in the lamina in agreement with the results by Lee et al. [9].

Changes in the solvent properties similar to the ones for pure water near smooth walls are found for a 2.2 molal LiI solution confined between two LJ walls. As an example for the structural changes Fig. 7 shows the $\mathrm{O}-\mathrm{O}, \mathrm{O}-\mathrm{H}$, and $\mathrm{H}-\mathrm{H}$ RDFs in the LiI solution[15]. The full lines are for reference atoms located in the surface layer while the dashed lines correspond to the central part of the lamina. The inner layer RDFs compare very well with those for the bulk solution [16]. The wall layer functions show a much more pronounced first neighbour shell than in bulk water. This leads to the conclusion that the LJ walls, which can be regarded as very large unpolar solutes, are short-ranging structure makers.

Figure 8 gives the anisotropic pair correlation function (PCF) $g_{\mathrm{OO}}\left(\Delta z, \Delta \varphi ; z_{\mathrm{O}}\right)$ for reference atoms

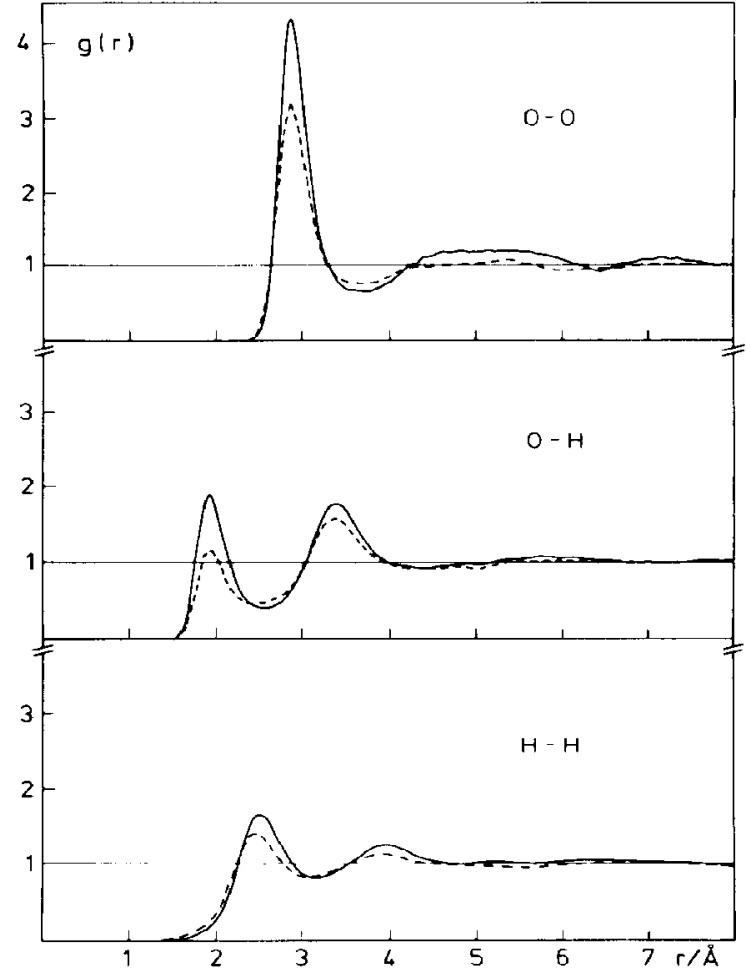

Fig. 7. Oxygen-oxygen, oxygen-hydrogen, and hydrogenhydrogen radial distribution functions for the water molecules in a $2.2 \mathrm{~m}$ LiI solution between $\mathrm{LJ}$ walls in the region close to the wall (full line) and in the interior of the lamina (dashed line)[15].

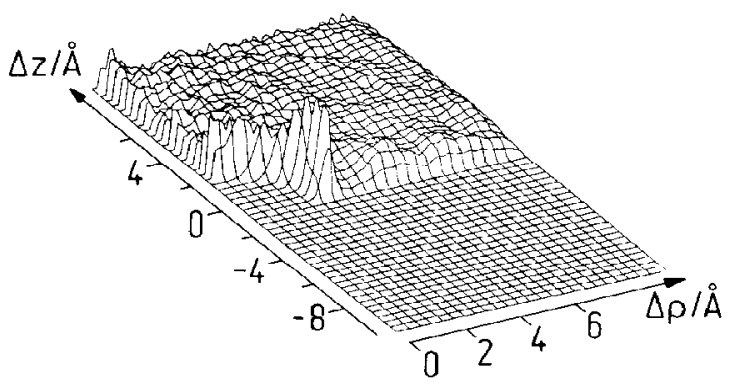

Fig. 8. Anisotropic oxygen-oxygen pair correlation function $g_{\mathrm{OO}}\left(\Delta z, \Delta \rho ; z_{\mathrm{O}}\right)$ for water molecules in the wall layer from a simulation of a $2.2 \mathrm{~m}$ Lil solution between Lennard--Jones walls. $\Delta z$ and $\Delta \rho$ are the components of the interparticle distance vector perpendicular and parallel to the surface, respectively[48].

close to the wall. $\Delta z$ is the projection of the oxygen-oxygen vector along the inward directed wall normal and $\Delta \rho$ is its projection parallel to the walls. The drawings clearly illustrate the origin of the increase in peak height for $g_{0 o}(r)$ (Fig. 7). The probability of finding a nearest neighbour is significantly larger in the direction parallel to the wall than 
perpendicular to it. Although the weak onedimensional LJ potential does not exhibit a direct ordering effect, the PCFs in the wall layer are compatible with a perturbed ice Ih like structure in agreement with the results found by Lee et al. [9].

Computer simulations indicate more dramatic changes in water structure near more realistic, structured surfaces than between smooth walls. A detailed discussion of the pair correlation functions for ST2 water near the $(100)$ surface of a platinum crystal is presented in[31]. A combination of $\mathrm{LJ}$ potentials between oxygen and platinum atoms with image charges has been used to describe the water-wall interactions. In spite of several shortcomings of that model further investigations with more sophisticated water-platinum interaction potentials have shown that the essential effect of the metal surface on the water PCFs is properly described. On the (100) surface the adsorption site arrangement is a quadratic grid with a spacing of $2.77 \AA$. This allows for hydrogen bonding between pairs but is not compatible with the local tetrahedral network formed in bulk water. In Figs 9-11 it is demonstrated how a complete structural picture can be derived from the molecular dynamics simulation. All pair correlation functions are calculated according to the definition:

$$
g\left(\mathbf{r}_{1}, \mathbf{r}_{2}\right)=\frac{\rho^{(2)}\left(\mathbf{r}_{1}, \mathbf{r}_{2}\right)}{\rho^{(1)}\left(\mathbf{r}_{1}\right) \rho^{(1)}\left(\mathbf{r}_{2}\right)},
$$

with $\rho^{(i)}$ the local $i$-particle density. Integrations over appropriate variables lead to the PCFs described below. Through use of the local 1-particle density the PCFs can be normalized properly to the limiting value of one for large distances [49].

Figure 9 shows the $z$-dependent RDFs $g_{O O}\left(r ; z_{1}\right)$, $g_{\mathrm{OH}}\left(r ; z_{1}\right)$, and $g_{\mathrm{HH}}\left(r ; z_{1}\right)$ for three subsystems. They have been calculated by selecting particle 1 from a certain range of $z_{1}$-values only and sampling distances to all other atoms of the specified kind in the lamina. The full curves are the RDFs around the adsorbed molecules $\left(\left|z_{1}\right|>7.5 \AA\right)$, the dotted lines those around the molecules situated in the second layer (5.6 $\AA<\left|z_{1}\right|$ $\leqslant 7.5 \AA$ ) and the dashed lines are the RDFs around the bulk like molecules in the center of the lamina $\left(\left|z_{1}\right|\right.$ $\leqslant 5.6 \AA$ ). The walls are positioned at $z= \pm 11.25 \AA$. The error bars given at the locations of the first maxima correspond to the difference in RDF between the left half and right half of the lamina. The center molecules (dashed) show essentially the behaviour of bulk water [6] apart from an increase of the height of the first maximum. This increase is due to the lower density in the center of the lamina than in bulk water and is consistent with the results of Geiger [50] for ST2 water at a density of about $0.7 \mathrm{~g} \mathrm{~cm}^{-3}$. The depletion in the center is a consequence of the adsorption of water molecules on the surface, since the system size has been fixed such that the average density in the lamina equals the bulk density of water (see also discussion of Fig. 5).

The layer of adsorbed water molecules (full curve) resembles the lattice structure beyond the first neighbour shell as can be seen from three new maxima occurring at about $4,5.5-6.3$, and $8.5 \AA$, which correspond roughly to $2^{1 / 2}, 2$ and $5^{1 / 2}$, and $8^{1 / 2}$ and 3 times the nearest neighbour distance, respectively. The position of the nearest neighbour peak does not

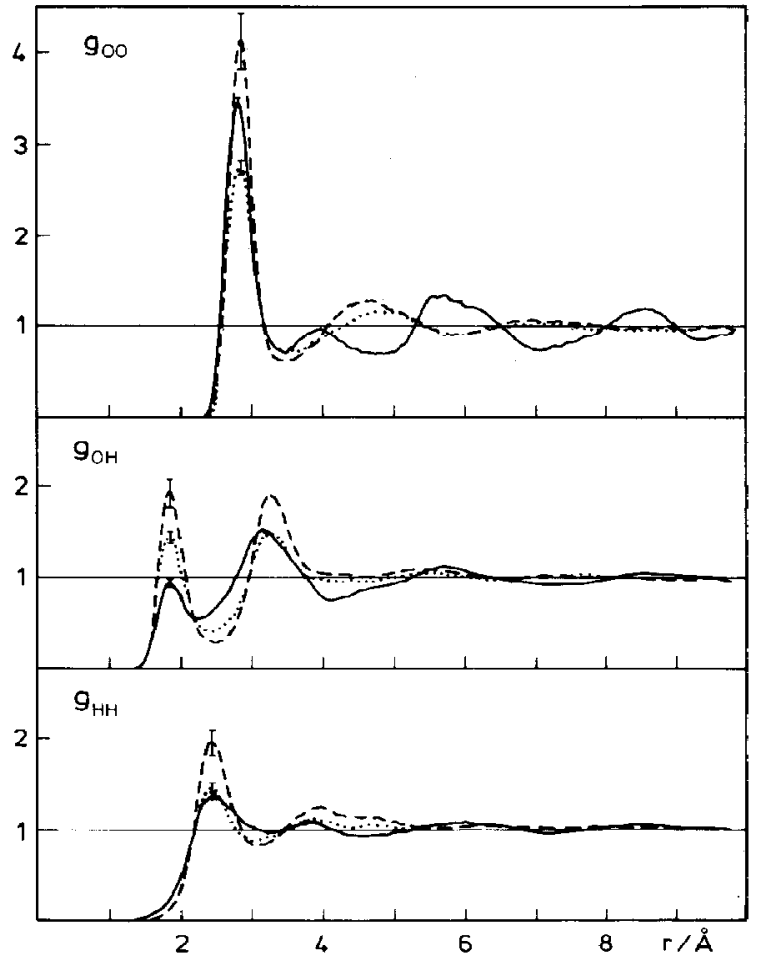

Fig. 9. Atom-atom radial distribution functions $g_{a \theta}\left(r ; z_{1}\right)$ $(\alpha, \beta=\mathrm{O}, \mathrm{H})$ for three subsystems (characterized by the coordinate $z_{1}$ ) from a simulation of ST2 water between the (100) faces of a platinum crystal: the adsorbate layer (full line; $\left|z_{1}\right|>7.5 \AA$ ), the second layer (dotted line; $5.6 \AA<\left|z_{1}\right|$ $\leqslant 7.5 \AA$ ), and the center of the lamina (dashed line; $\left|z_{1}\right|$ $\leqslant 5.6 \AA$ ). The walls are positioned at $z= \pm 11.25 \AA[31]$.

change compared to bulk water in accordance with the similarity of the lattice periodicity $(2.77 \AA)$ and the average water-water distance $(2.85 \AA)$ in bulk water. In the intermediate layer the transition from the quadratic surface structure to the normal tetrahedral short range order obviously leads to a perturbation of water structure as is indicated by the low first maximum in $g_{\text {oo }}(r)$.

The adsorption leads to a significant reduction of the height of the first intermolecular peak in the oxygen-hydrogen and hydrogen-hydrogen RDFs (Fig. 9), indicating weaker hydrogen bonds on the quadratic substrate lattice than in the bulk. The second maximum of $g_{\mathrm{OH}}(r)$ is shifted by more than $0.1 \AA$ to smaller distances and the minimum between the two maxima is considerably shallower. This effect is again due to the quadratic arrangement of the adsorbed molecules that leads with high probability to hydrogen bonds pointing along the two surface diagonals $x=y$ and $x=-y$. An ST2 molecule (HOH angle $=109.47^{\circ}$ ), with its dipole moment parallel to the surface, can form only one linear bond at a time and hence the first and second maximum is reduced in height. While in a tetrahedral arrangement the oxygen-second hydrogen distance is $\approx 3.3 \AA$, the 
corresponding distance is around $3.0 \AA$ for the quadratic network. The observed value lies between these bounds. In the intermediate layer the first peak is still lower than in bulk water and the second peak is found at the bulk distance of $3.3 \AA$. The central part of the lamina shows pronounced maxima and a very deep minimum as a consequence of the increased tetrahedral short range order compared to bulk water at a density of $1 \mathrm{~g} \mathrm{~cm}^{-3}$. The perturbation of short range order close to the interface can also be seen in the reduced peak heights of the hydrogen-hydrogen RDF.

Figure 10 demonstrates the degree of anisotropy of the oxygen-oxygen pair correlation functions in the center of the lamina (a) and close to the wall (b) in the same manner as Fig. 8 . The data had to be smoothed before plotting in order to remove statistical noise. As a consequence, the heights, especially of the first neighbour peaks, are significantly reduced. The peak positions, however, remain unaffected. Fig. 10b shows that the changes of $g_{\mathrm{OO}}\left(r ; z_{1}\right)$ (Fig. 9) beyond the first neighbor shell are due solely to correlations parallel to the surface, which show the characteristic far reaching oscillations. One also infers from Fig. $10 \mathrm{~b}$ that the probability of finding a nearest neighbor in the surface plane is considerably higher than perpendicular to the surface. In spite of the relative to the LJ walls much stronger interactions, the oxygen-oxygen PCF is practically isotropic in the center of the lamina as can be seen from Fig. 10a.

To obtain a closer view of the correlations between adsorbed molecules, the PCFs $g_{O O}\left(|\Delta x|,|\Delta y| ; z_{1}, z_{2}\right)$ are plotted in Fig. 11. $\Delta x$ and $\Delta y$ are the components of the interparticle vector along the two laboratory frame axes parallel to the surface (the $(001)$ and $(010)$ directions of the crystal, respectively; cf. Fig. 1) and both atoms are located in the adsorbed layer $\left(\left|z_{1}\right|,\left|z_{2}\right|\right.$ $>7.5 \AA$ ). This figure demonstrates directly what has been deduced already from Figs 9 and 10, namely that

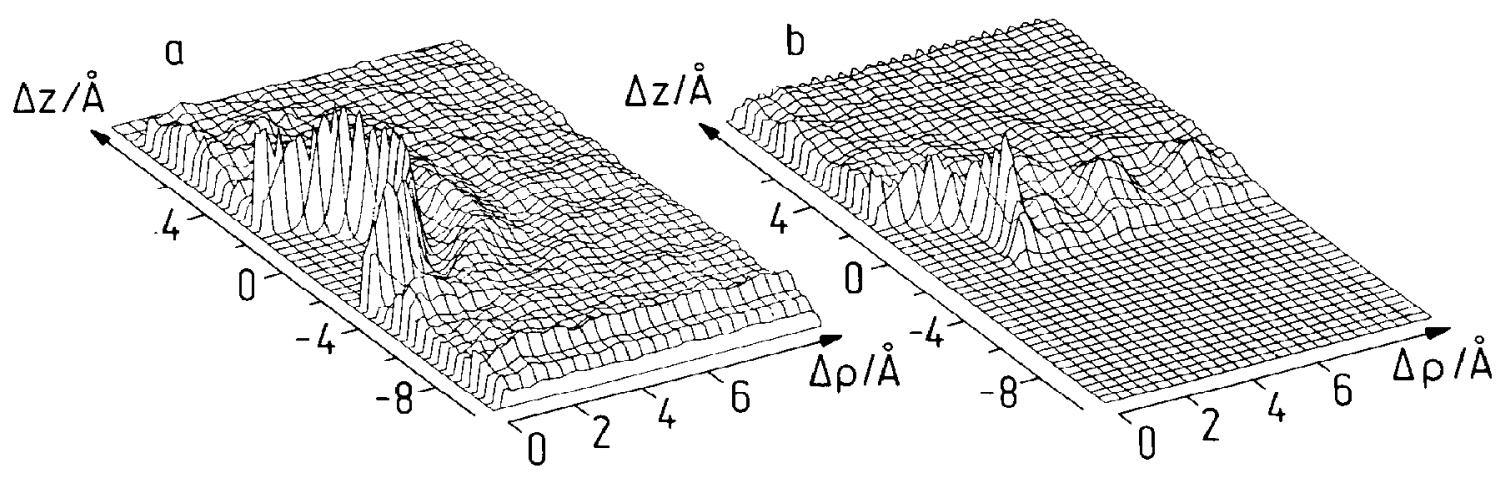

Fig. 10. Anisotropic oxygen-oxygen pair correlation function $g_{\mathrm{OO}}\left(\Delta z, \Delta \rho ; z_{\mathrm{O}}\right)$ for water molecules in the central part of the lamina (a) and the wall layer (b) from a simulation of ST 2 water between the (100) faces of a platinum crystal (see also caption of Fig. 8)[31].

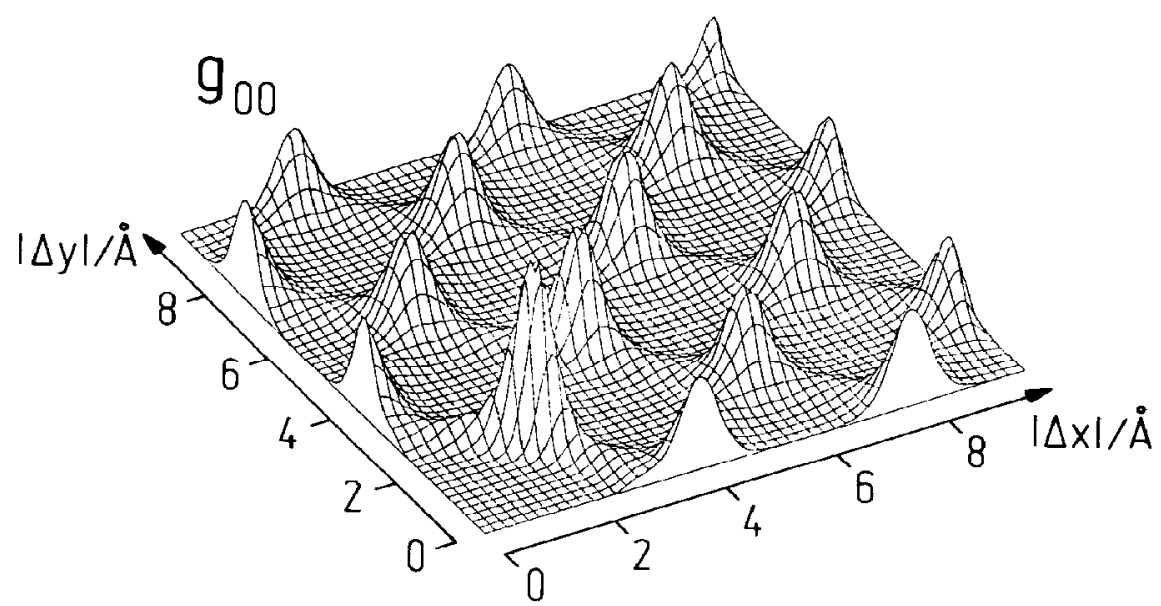

Fig. 11. Oxygen-oxygen pair correlation function $g_{O O}\left(|\Delta x|,|\Delta y| ; z_{1}, z_{2}\right)$ between adsorbed water molecules only, from a simulation of ST2 water between the $(100)$ faces of a platinum crystal. $\Delta x$ and $\Delta y$ are the projections of the interparticle distance vector onto the $x$ - and $y$-directions of the laboratory coordinate system, respectively, and $\left|z_{1}\right|,\left|z_{2}\right|>7.5 \AA$ (see also caption of Fig. 9)[31]. 


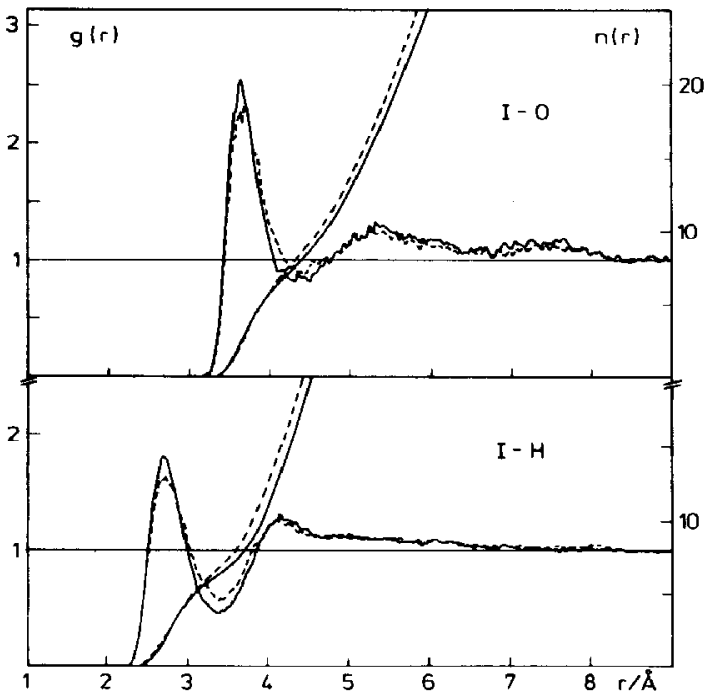

Fig. 12. Iodide-oxygen and iodide-hydrogen radial distribution functions $g(r)$ and running integration numbers $n(r)$ for the $2.2 \mathrm{~m} \mathrm{LiI} \mathrm{solution} \mathrm{confined} \mathrm{between} \mathrm{two} \mathrm{LJ} \mathrm{walls} \mathrm{(full}$ lines) and for a bulk $2.2 \mathrm{~m} \mathrm{Lil}$ solution (dashed lines)[15].

the oxygen atoms of the water molecules form a quadratic overlayer commensurate with the crystal periodicity which necessarily results in far reaching oxygen-oxygen correlations [31].

The influence of an interfacial inhomogeneity on the $\mathrm{Li}^{+}$and $\mathrm{I}^{-}$hydration shells has also been investigated in a simulation of a 2.2 molal LiI solution between $\mathrm{LJ}$ walls. It has been found that the $\mathrm{Li}^{+}$hydration shell remains unchanged[15]. This is the expected result as the ion-wall and water-wall interactions are very weak compared to the strong hydration forces of the $\mathrm{Li}^{+}$.

However, significant changes in $g_{I J}(r)$ and $h_{I I I}(r)$ can be detected in the lamina. The functions and the running coordination numbers are depicted in Fig. 12 (full lines) together with the corresponding ones from a bulk simulation by Szász and Heinzinger[16] (dashed lines). Due to the steric effect of the walls the running integration numbers are always lower in the lamina yielding a hydration number of 7.7 (from the value of $n_{\text {Io }}$ at the location of the first minimum) which has to be compared to 8.7 in the isotropic solution. Both RDFs cxhibit a higher first maximum and a lower first minimum for both $g_{\mathrm{IO}}(r)$ and $g_{\mathrm{IH}}(r)$ in the lamina. Additionally, if the distance at which $g_{10}(r)$ equals 1 for the second time is taken as a measure of the radial localiztion of the hydration shell it is significantly narrower in this work than in the isotropic solution thus indicating an enhancement of hydration shell structure around the iodide ion. At present, further studies of the hydration structure of ions near metal surfaces and of the extent to which contact adsorption of large anions occurs are under way.

The pair correlation functions of water discussed in this chapter still suffer from statistical uncertainty. To investigate the structural and dynamic properties of electrolyte solutions between metal walls with a reasonable degree of reliability the simulation times should be significantly longer and the box sizes larger than for pure water.

\section{DYNAMICAL PROPERTIES}

In most of the simulations reported so far the MC method has been used. Therefore, only a few authors deal with dynamical properties of water at interfaces. Marchesi et al. $[5,8]$ have calculated the self-diffusion coefficient of water from the mean square displacements:

$$
D=\lim _{t \rightarrow \infty}\left\langle[\mathbf{R}(t)-\mathbf{R}(0)]^{2}\right\rangle / 6 t,
$$

and also the dipole relaxation time:

$$
\tau_{1}=\int_{0}^{x} \Gamma_{1}(t) \mathrm{d} t
$$

with the correlation function:

$$
\Gamma_{1}(t)=\left\langle\mu_{j}(0) \mu_{j}(t)\right\rangle
$$

where $\mu_{j}(t)$ is the unit vector in the dipole moment direction of water molecule $j$ at time $t$.

For ST2 water between smooth $L J[7,15]$ and corrugated platinum [49] walls the self-diffusion coefficients have been calculated from the simulations through the Green-Kubo relation:

$$
D=\lim _{t \rightarrow \infty} \int_{0}^{t} c_{v}(t) \mathrm{d} t,
$$

with the velocity autocorrelation function (vacf):

$$
c_{v}(t)=\frac{1}{N_{T} N} \sum_{i=1}^{N_{T}} \sum_{j=1}^{N} v_{j}\left(t_{i}\right) v_{j}\left(t_{i}+t\right),
$$

where $N$ denotes the number of particles, $N_{T}$ the number of time averages and $v_{j}(t)$ the velocity of particle $j$ at time $t$. The spectral densities of hindered translations and librations have been calculated by Fourier transformation from the velocity and angular velocity autocorrelation functions.

Marchesi et al. have found an increase of the dipolar relaxation time in the wall layer of up to $65 \%$ relative to bulk water. The increase depends on the water-wall potential employed. The authors point out that there remains quite some doubt on the quantitative significance of these data because of the large statistical noise $[5,8]$.

The center of mass vacfs parallel and perpendicular to the interface $\left(c_{v_{x y}}\right.$ and $c_{v_{x}}$ ) for the wall layer (full line) and the central layer of water molecules in the lamina (dash-dotted line) are depicted in Fig. 13 for $\mathrm{LJ}$ walls $[7,15]$ and in Fig. 14 for platinum walls [49]. The spectral densities of the corresponding hindered translational motions are given in the insertions. Both figures show that the shape of vacfs in the centre of the lamina is practically identical for the motions parallel and perpendicular to the interface for the two different systems. This indicates that changes in dynamical properties, induced by the interfacial interactions, are short ranged, similar to what has been found for the water structure. The weaker binding energy of a water molecule near the $L J$ wall influences the dynamical behaviour in that way that $c_{v_{x y}}$ does not pass through zero at about 0.07 ps (Fig. 13). Different from the bulk, 


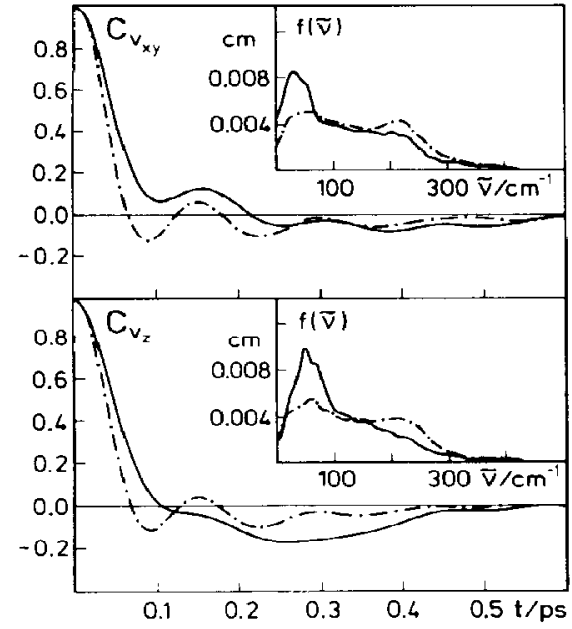

Fig. 13. Velocity autocorrelation functions and the spectral densities of hindered translational motions (insertions) parallel $\left(c_{v_{x y}}\right)$ and perpendicular to the wall $\left(c_{v_{z}}\right)$ for water molecules close to the LJ walls (full lines) and in the interior of a lamina (dashed lines)[15].

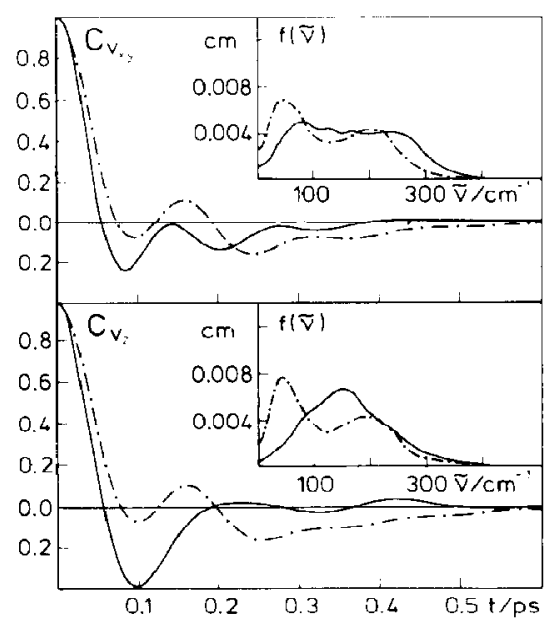

Fig. 14. Velocity autocorrelation functions and the spectral densities of hindered translational motions (insertions) parallel $\left(c_{v_{x y}}\right)$ and perpendicular to the wall $\left(c_{v_{x}}\right)$ for water molecules close to the (100) faces of a platinum crystal (full lines) and in the interior of a lamina (dashed lines)[49].

the direction of the motion does not change after that time.

The spectral densities of the hindered translational motions of the water molecules between smooth and non-polar LJ walls (insertions in Fig. 13) show a strong increase at low frequencies around $50 \mathrm{~cm}^{-1}$ and a decrease around about $200 \mathrm{~cm}^{-1}$ for the molecules close to the wall. The effect is quite similar for motions parallel and perpendicular to the wall. The frequencies around $50 \mathrm{~cm}^{-1}$ are usually assigned to the $\mathrm{O}-\mathrm{O}-\mathrm{O}$ bending motion [51] and rotational modes of $\left(\mathrm{H}_{2} \mathrm{O}\right)_{3}$ complexes (for the analysis of these modes see[52]).
Obviously, due to the more pronounced tetrahedral short range order near the non-polar interfaces $[7,9]$ bend type motions in which one molecule moves through the gap between two other molecules are favoured. This conclusion is supported by the reverse effect found in an MD simulation of high density water where the intensity of the $\mathrm{O}-\mathrm{O}-\mathrm{O}$ bending mode is reduced relative to water at normal density [53].

For water near platinum crystals not only the relative contributions of the different modes change but also new modes develop because of the much stronger water-wall interactions when compared with LJ walls (insertions in Fig. 14). Perpendicular to the surface a new band at about $150 \mathrm{~cm}^{-1}$ appears which is due to the hindered translation of the water molecule against the surface. Parallel to the surface the power spectrum shows a broad band between about 80 and $270 \mathrm{~cm}^{-1}$ with a significant increase of high and decrease of low frequency contributions.

The normalized angular velocity autocorrelation functions have been calculated. separately for the principal rotational axes of the water molecule $\left(c_{\omega_{i}}\right)$ as well as for the molecules close to the walls (full) and in the interior of the lamina (dashed). They are depicted in Fig. 15 together with the corresponding spectral

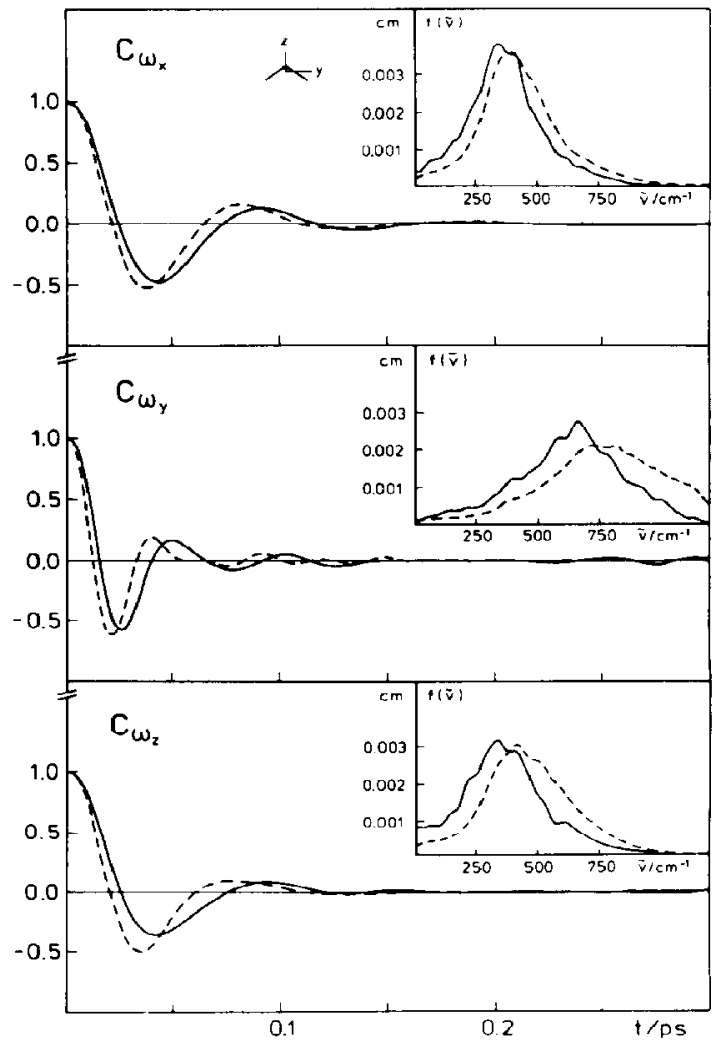

Fig. 15. Angular velocity autocorrelation functions and spectral densities of librational motions of water molecules for rotations about the three principal molecular axes (insertions) for water molecules close to LJ walls (full lines) and in the interior of a lamina (dashed lines)[15]. 
densities of the librations (insertions) for water between LJ walls[15]. All rotations about the principal axes of the water molecules close to the wall show a tendency to preserve their motions for longer times than in the inner part of the lamina as can be seen from the shift of the first zeros of the autocorrelation functions to longer times. Accordingly, the spectral densities of the librations (insertions in Fig. 15) are shifted by about $60-100 \mathrm{~cm}^{-1}$ to lower wavenumbers. This shift results from the reduced number of hydrogen bonds formed by the water molecules in the wall layer. The simulations for the platinum walls lead to qualitatively similar results as for $L J$ walls as the number of hydrogen bonds is also reduced near the walls. Therefore, these $c_{w_{i}}$ are not shown here.

Spectroscopic investigations of water adsorbed on a platinum (100) surface at $150 \mathrm{~K}$ lead to frequencies for hindered translations of $240 \mathrm{~cm}^{-1}$, for the metal-water vibration of $460 \mathrm{~cm}^{-1}$, and for librations of 560 and $920 \mathrm{~cm}^{-1}[54,55]$. The comparison with the results from the simulation shows that the hindered translations parallel to the surface are described reasonably well which means that the height of the proposed barrier for surface diffusion in the model seems to have the right order of magnitude. The frequency for the motion of a water molecule perpendicular to the surface and the librational frequencies presented in Figs 14 and 15 are generally lower than the measured ones. The differences between simulation and experiment seem to result either from the fact that the additional water-water interactions in the model system reduce the frequencies or that the water-wall interaction potential is still not steep enough although it leads to a sharp maximum in the oxygen atom density profile for the adsorbed layer $\left(\rho / \rho_{0} \approx 7\right.$ in Fig. 5$)$.

Marchesi et al. performed various simulations with ST2 and MCY water and several water-wall potentials and found in all cases that the self-diffusion coefficient of water in the wall layer is by about $20 \%$ smaller than in the interior of the lamina $[5,8]$. They have calculated separately the self-diffusion coefficients parallel and perpendicular to the wall and showed that the difference of $20 \%$ results solely from a decrease of the mobility perpendicular to the wall. But these data are not reliable as it has been demonstrated by Sonnenschein and Heinzinger[7] that the selfdiffusion coefficient perpendicular to the wall cannot be calculated correctly from the mean square displacement. These authors showed that a residence autocorrelation function should be used with proper boundary conditions. With their method they found for water close to $L J$ walls an increase in the self-diffusion coefficient of about $50 \%$ relative to the interior of the lamina, both for the motion parallel and perpendicular to the interface. The increased mobility near the surface can be understood qualitatively on the basis of an approximately $25 \%$ decrease in the number of hydrogen bonds which is not completely balanced by the increase in average bond strength. The mobility of the water molecules near a platinum surface is strongly reduced. A self-diffusion coefficient can, therefore, not be calculated reliably.

As the comparison with experiment leads to qualitative agreement for the hindered translational frequencies parallel to the platinum surface, it seems appropri-

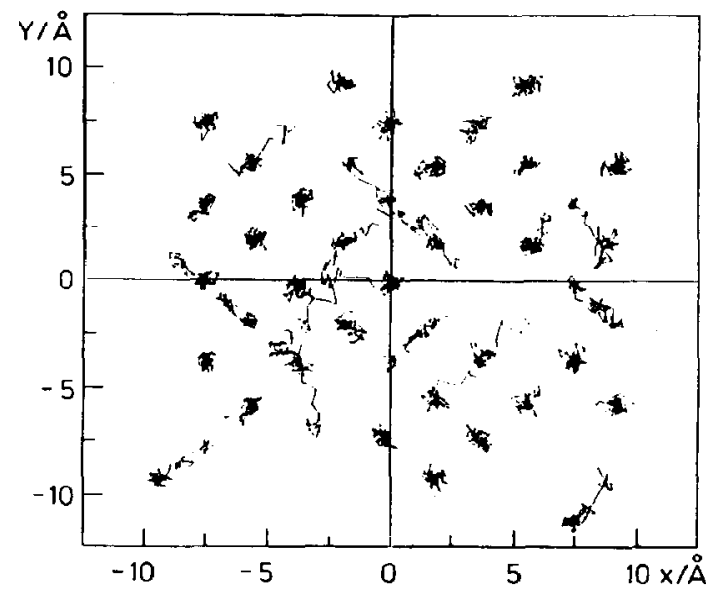

Fig. 16. Projection of the trajectories of water molecules adsorbed on the platinum (100) surface.

ate to elucidate this motion in some more detail. Figure 16 shows the trajectories of the adsorbed water molecules projected onto the interfacial plane for a time interval of $10 \mathrm{ps}$. It is obvious that the diffusional motion is jump-like with long intermediate periods of oscillations in the adsorption well. Furthermore, the existence of vacancies is recognized. Vacancies seem to be a necessary condition for rendering possible a jump between two sites, as the long stretched parts of the trajectories are seen only in the vicinity of these vacancies. A considerable amount of water molecules do not change sites on a time scale of $10 \mathrm{ps}$.

\section{CONCLUDING REMARKS}

The simulations of water and electrolyte solutions at interfaces reported so far have, in our opinion, demonstrated that computer simulations can be a useful tool in electrochemistry. It is also obvious that the potentials describing the water-wall interactions need to be improved significantly. Additionally, the simulations have to be extended as far as the system size and simulation time is concerned. With these improvements quantitative agreement with experimental data can be expected. Once this agreement is achieved properties which cannot or not directly be measured can be predicted reliably. The computer simulations can also contribute to an understanding of measured electrochemical properties on a molecular level.

Acknowledgement-Financial support by Deutsche Forschungsgemeinschaft is gratefully acknowledged.

\section{REFERENCES}

1. B. Jönsson, Chem. Phys. Lett. 82, 520 (1981).

2. O. Matsuoka, E. Clementi and M. Yoshimine, J. chem. Phys. 64, 1351 (1976).

3. N. I. Christou, J. S. Whitehouse, D. Nicholson and N. G. Parsonage, Faraday Symp. Chem. Soc. 16, 139 (1981). 
4. J. S. Rowlinson, Trans. Faraday Soc. 47, 120 (1951).

5. M. Marchesi, Chem. Phys. Letr. 97, 224 (1983).

6. F. H. Stillinger and A. Rahman, J. chem. Phys. 60, 1545 (1974).

7. R. Sonnenschein and K. Heinzinger, Chem. Phys. Lett. 102, 550 (1983).

8. G. Barabino, C. Gavotti and M. Marchesi, Chem. Phys. Lett. 104, 478 (1984).

9. C. Y. Lee, J. A. McCammon and P. J. Rossky, J. chem. Phys. 80, 4448 (1984).

10. N. I. Christou, J. S. Whitehouse, D. Nicholson and N. G. Parsonage, Mol. Phys. 55, 397 (1985).

11. W. L. Jorgensen, J. chem. Phys. 77, 4156 (1982).

12. G. Aloisi, R. Guidelli, R. A. Jackson, S. M. Clark and P. Barnes, J. electroanal. Chem. 206, 131 (1986).

13. J. P. Valleau and A. A. Gardner, I. chem. Phys. 86, 4162 (1987).

14. P. Linse, J. chem. Phys. 86, 4177 (1987).

15. E. Spohr and K. Heinzinger, J. chem. Phys. 84, 2304 (1986).

16. Gy. I. Szász, K. Heinzinger and W. O. Riede, $Z$. Naturforsch. 36, 1067 (1981).

17. Gy. I. Szász, K. Heinzinger and W. O. Riede, Ber Bunsenges. Phys. Chem. 85, 1056 (1981).

18. Gy. I. Szász and K. Heinzinger, J. chem. Phys. 79, 3467 (1983).

19. N. Anastasiou, D. Fincham and K. Singer, I. Chem Soc. Faraday Trans. 79, 1639 (1983).

20. F. H. Stillinger and A. Rahman, J. chem. Phys. 68, 666 (1978).

21. R. Kjellander and S. Marcelja, Chemicu Scripta 25, 73 (1985).

22. R. Kjellander and S. Marcelja, Chem. Phys. Lett. 120, 393 (1985).

23. E. Spohr and K. Heinzinger, Chem. Phys. Lett. 123, 218 (1986).

24. N. G. Parsonage and D. Nicholson, J. chem. Soc. Faraday Trans. 82, 1521 (1986).

25. N. G. Parsonage and D. Nicholson, J. Chem. Soc. Faraday Trans. 83, 663 (1987).

26. A. A. Gardner and J. P. Valleau, J. chem. Phys. 86, 4171 (1987).

27. A. Luzar, D. Bratko and L. Blum, J. chem. Phys. 86, 2955 (1987).

28. J. Eggebrecht, S. M. Thompson and K. E. Gubbins, $J$. chem. Phys. 86, 2299 (1987).
29. S. L. Carnie, Ber. Bunsenges. Phys. Chem. 91,262 (1987).

30. M. L. Rosinberg, V. Russier, J. P. Badiali and M. E. Boudh'hir, Ber. Bunsenges. Phys. Chem. 91, 276 (1987).

31. E. Spohr, to be published.

32. Th. E. Madey and J. T. Yates Jr, Chem. Phys. Lett. 51, 77 (1977).

33. G. B. Fisher and J. L. Gland, Sturf. Sci. 94, 446 (1980).

34. C. Benndorf, C. Nöbl and F. Thieme, Surf. Sci. 121, 249 (1982).

35. J. Fusy and R. Ducros, Surf. Sci. 176, 157 (1986).

36. E. Sophr and G. Pálinkás, in Interactions of Water in Ionic and Nonionic Hydrates, (Edited by H. Kleeberg), Springer, Berlin, 221 (1987)

37. E. Spohr, to be published.

38. P. Bopp, G. Jancsó and K. Heinzinger, Chem. Phys. Lett. 98, 129 (1983).

39. S. Holloway and K. H. Bennemann, Surf. Sci. 101, 327 (1980).

40. C. W. Bauschlicher Jr, J. chem. Phys. 83, 3129 (1985).

41. M. W. Ribarsky, W. D. Luedtke and U. Landman, Phys. Rev. 32, 1430 (1985).

42. J. Müller, Surf. Sci. 178, 589 (1986).

43. E. Langenbach, A. Spitzer and H. Lüth, Surf. Sci. 147, 179 (1984).

44. D. E. Peebles and J. M. White, Surf. Sci. 144, 512 (1984).

45. K. Bange, T. E. Madey, J. K. Sass and E. M. Stuve, Surf. Sci. 183, 334 (1987).

46. C. Mariani and K. Horn, Surf. Sci. 126, 279 (1983)

47. K. Bange, D. E. Grider, T. E. Madey and J. K. Sass, Surf. Sci. 136, 38 (1984).

48. R. Sonnenschein, Ph.D. Thesis, Johannes-GutenbergUniversität, Mainz (1984).

49. E. Spohr, Ph.D. Thesis, Johannes-GutenbergUniversität, Mainz (1986).

50. A. Geiger, P. Mausbach and J. Schnitker, in Water \& Aqueous Solutions, Proceedings of the Thirty-seventh Symposium of the Colston Research Society, (Edited by G. W. Neilson and J. E. Enderby), Adam Hilger, Bristol and Boston, 15 (1985).

51. M. G. Sceats and S. A. Rice, J. chem. Phys. 72, 3236 (1980).

52. E. Spohr and P. Bopp, to be published.

53. G. Janscó, P. Bopp and K. Heinzinger, Chem. Phys. 85, 377 (1984).

54. H. Ibach and S. Lehwald, Surf. Sci. 91, 187 (1980)

55. J. E. Crowell, J. G. Chen, D. M. Hercules, J. T. Yates Jr, J chem. Phys. 86, 5804 (1987). 